Received: 20 September 2018 Accepted: 11 June 2019 Published online: 26 June 2019
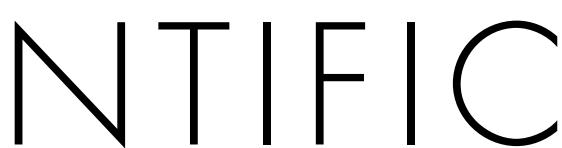

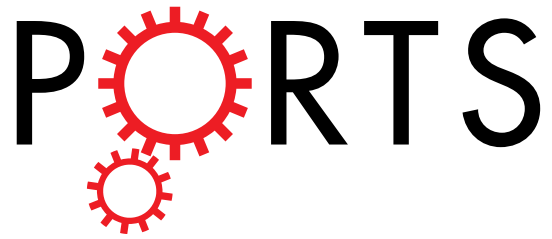
transformed A Body Shape Index (LBSIZ) in the assessment for sarcopenic obesity and cardiovascular disease risk in the United States

\author{
Wankyo Chung ${ }^{1,2}$, Jung Hwan Park ${ }^{3}$, Hye Soo Chung ${ }^{4}$, Jae Myung Yu ${ }^{4}$, Dong Sun Kim ${ }^{3} \&$ \\ Shinje Moon ${ }^{4,5}$
}

Body mass index (BMI) has limited accuracy for predicting cardiovascular diseases (CVD) and is not capable of identifying sarcopenic obesity, the combination of sarcopenia (an age-associated decline in muscle mass and physical function) and obesity. To overcome this, the z-score of the log-transformed A Body Shape Index (LBSIZ) was recently introduced as a measure of obesity using waist circumference, height, and weight. We aimed to investigate the association of LBSIZ with sarcopenic obesity and CVD, and propose appropriate cut-off values using the National Health and Nutrition Examination Survey 1999-2016 data. Of 92,062 participants, 40,468 adults ( $\geq 20$ years) were included. Overall area under curve (AUC) of LBSIZ was 0.735 ( $95 \%$ confidence interval [CI]: $0.716-0.754$ ) for sarcopenic obesity, and 0.695 (95\% Cl: $0.687-0.703$ ) for CVD. The subgroup analysis of ethnicity/race showed similar results. Waist circumference (WC), BMI, conicity index, body roundness index (BRI), Clinica Universidad de Navarra-Body Adiposity Estimator (CUN-BAE), new BMI, and waist to height ratio (WHtR) showed a negative association with sarcopenic obesity, while LBSIZ and conicity index showed a positive association. The AUC of LBSIZ was significantly higher for sarcopenic obesity than that of conicity index $(p<0.001)$. The AUC of LBSIZ was significantly higher for CVD than those of parameters including WC, BMI, BRI, CUN-BAE, new BMI, and WHtR $(p<0.001)$. The AUC for conicity index alone was comparable to that of LBSIZ for CVD. Overall LBSIZ cut-off was 0.35 for both sarcopenic obesity (sensitivity, $65.3 \%$; specificity, $71.5 \%$ ) and CVD (sensitivity, $63.3 \%$; specificity, $66.6 \%$ ). These results may be useful not only to identify sarcopenic obesity, but also to conduct CVD risk assessment in the clinical setting.

According to the World Health Organization (WHO), 13\% of the world population were estimated to be obese in 2016, the prevalence of which increased three times in the last three decades ${ }^{1}$. Obesity increases the risk of chronic diseases, such as cardiovascular disease (CVD), diabetes, stroke, and cancer, which are responsible for approximately $4.8 \%$ of worldwide death $\mathrm{s}^{2-6}$. The increasing prevalence of obesity and serious obesity-related diseases has made obesity a major public health concern. Another concern with aging population is sarcopenic obesity, which is the combined state of sarcopenia and obesity ${ }^{7}$. Sarcopenia is defined as the age-associated decline

${ }^{1}$ Department of Public Health Science, Graduate School of Public Health, Seoul National University, Seoul, Republic of Korea. ${ }^{2}$ Institute of Health and Environment, Seoul National University, Seoul, Republic of Korea. ${ }^{3}$ Department of Internal Medicine, Hanyang University College of Medicine, Seoul, Republic of Korea. ${ }^{4}$ Division of Endocrinology and Metabolism, Hallym University College of Medicine, Chuncheon, Republic of Korea. ${ }^{5}$ Department of Internal medicine, Graduate School, Hanyang University, Seoul, Republic of Korea. Correspondence and requests for materials should be addressed to D.S.K. (email: dongsun@hanyang.ac.kr) or S.M. (email: sinjei1129@gmail.com) 


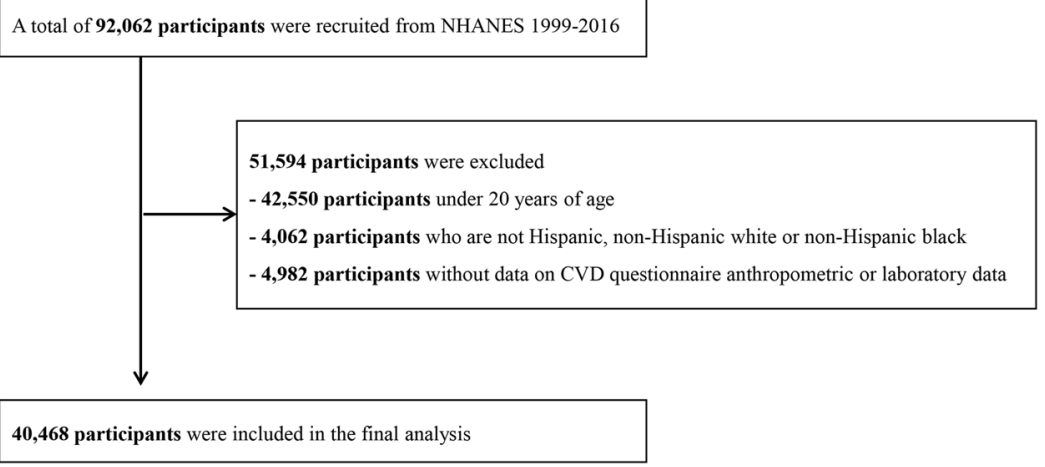

Figure 1. Flowchart showing the final selection. NHANES, National Health and Nutrition Examination Survey; CVD, cardiovascular disease.

in muscle mass and physical function, and may synergistically worsen the adverse effects of obesity, leading to higher disability, morbidity and mortality ${ }^{7}$.

Accurate assessment of obesity is required for the prevention and treatment thereof. Obesity can be assessed by directly measuring body fat via computed tomography (CT), magnetic resonance imaging, DEXA, and positron emission tomography (PET)-CT $\mathrm{CT}^{8}$. However, these methods are costly and have limitations that make the use of these modalities for diagnosing obesity in real clinical settings challenging; instead, indirect indices of obesity are used. Body mass index (BMI) has long been used, as it is easy to measure and calculate. However, BMI has limited accuracy for predicting the amount and distribution of body fat and is not capable of identifying sarcopenic obesity ${ }^{9}$. It is also limited in its ability to clinically predict the risk of chronic diseases such as CVD ${ }^{10-12}$. To overcome these limitations, A Body Shape Index (ABSI), which is a formula that uses waist circumference (WC), height, and weight, has recently been introduced ${ }^{13}$. However, ABSI has limited clinical usefulness due to not having cut-off points for identifying individuals at high risk for obesity-related diseases ${ }^{14,15}$. Therefore, we have previously proposed the z-score of the log-transformed ABSI (LBSIZ), which overcomes the limitations of the ABSI, using representative samples from Korea ${ }^{15-17}$. However, data is lacking in terms of its assessed usefulness in other populations.

Therefore, this study aimed to propose a LBSIZ formula for each race, using representative samples from the United States (US). We then examined its relationship with both sarcopenic obesity and CVD risk, compared to other obesity parameters, and provided appropriate cut-off values to identify individuals at high risk for sarcopenic obesity and CVD.

\section{Results}

Baseline characteristics. A total of 40,468 healthy adults (19,508 men and 20,960 women) from the US, aged $20-85$ years (mean age $=49.2$ ), were analyzed (Fig. 1 ). The anthropometric, clinical, and biochemical characteristics of the participants are summarized in Table 1 according to ethnicity/race. A total of 4,327 (10.7\%) participants had CVD (angina pectoris: 2.9\%; coronary heart disease: $4.1 \%$; myocardial infarction: $4.2 \%$; congestive heart failure: $3.1 \%$; cerebrovascular disease: $3.4 \%$ ). The distributions of LBSIZ according to ethnicity/race are summarized in Table 2 .

Association between obesity parameters and body composition. LBSIZ showed a positive association with fat mass index (FMI) and a negative association with appendicular skeletal mass index (ASMI), while all other obesity parameters showed a positive association with both FMI and ASMI (Table 3). Figure 2a shows the ROC curves for sarcopenic obesity according to obesity parameters. The overall Area Under the Curve (AUC) of LBSIZ for sarcopenic obesity was 0.735 (95\% confidence interval [CI]: 0.716-0.754). Other obesity parameters showed a negative association with sarcopenic obesity, while LBSIZ and conicity index showed a positive association. The AUC of LBSIZ was significantly higher than that of conicity index ( $p<0.001)$. In the subgroup analysis according to ethnicity/race, the AUCs for sarcopenic obesity were 0.717 (95\% CI: $0.681-0.753$ ), 0.740 (95\% CI: 0.716-0.764), and 0.804 (95\% CI: 0.733-0.876) for the Hispanic, Non-Hispanic white, and Non-Hispanic black groups, respectively. The overall cut-off value of LBSIZ for sarcopenic obesity was $0.35\left(65^{\text {th }}\right.$ percentile, sensitivity, $65.3 \%$; specificity, $71.5 \%$ ). The corresponding sensitivity and specificities by ethnicity/race were $65.1 \%$ and $70.9 \%$, $64 \%$ and $62.3 \%$, and $81.8 \%$ and $60.6 \%$ for the Hispanic, Non-Hispanic white, and Non-Hispanic black groups, respectively. The OR for sarcopenic obesity adjusted for age, sex, and ethnicity/race was 2.38 (95\% CI, 2.05-2.77) at the estimated cut-off value of LBSIZ. On the restricted cubic spline regression plot, ORs increased after the median value (0) of LBSIZ (Fig. 3b)

Association between obesity parameters and CVD. Figure $2 \mathrm{~b}$ shows the ROC curves for CVD according to the obesity parameters. The overall AUC of LBSIZ for CVD was 0.695 (95\% CI: 0.687-0.703), which was significantly higher than those of WC ( $p$ value $<0.001)$, BMI ( $p$ value $<0.001$ ), Body Roundness Index (BRI) $(\mathrm{p}<0.001)$, Clinica Universidad de Navarra-Body Adiposity Estimator (CUN-BAE) $(\mathrm{p}<0.001)$, New BMI $(\mathrm{p}<0.001)$ and waist to height ratio (WHtR) $(\mathrm{p}<0.001)$. Conicity index alone showed a comparable AUC to that of LBSIZ. The bootstrap's bias corrected $95 \%$ CI of AUC of LBSIZ was $0.687-0.703$. In the subgroup analysis 


\begin{tabular}{|c|c|c|c|c|c|}
\hline \multirow[b]{2}{*}{ Variable } & \multicolumn{4}{|l|}{ Ethnicity/race } & \multirow[b]{2}{*}{ Total } \\
\hline & $\begin{array}{l}\text { Hispanic } \\
(\mathrm{N}=11596)\end{array}$ & $\begin{array}{l}\text { Non-Hispanic White } \\
(\mathrm{N}=19815)\end{array}$ & $\begin{array}{l}\text { Non-Hispanic Black } \\
(\mathrm{N}=9057)\end{array}$ & $P$ & \\
\hline Age (years) & $46.3 \pm 16.9$ & $51.6 \pm 19.0$ & $47.7 \pm 17.0$ & $<0.001$ & $49.2 \pm 18.1$ \\
\hline Men & $5423(46.8 \%)$ & $9722(49.1 \%)$ & $4363(48.2 \%)$ & $<0.001$ & $19508(48.2 \%)$ \\
\hline Smoking ( $\geq 100$ cigarettes in life) & $4539(39.2 \%)$ & $10522(53.1 \%)$ & $3960(43.8 \%)$ & $<0.001$ & $19021(47.0 \%)$ \\
\hline $\operatorname{BMI}\left(\mathrm{kg} / \mathrm{m}^{2}\right)$ & $29.3 \pm 6.0$ & $28.3 \pm 6.4$ & $30.2 \pm 7.5$ & 0.002 & $29.0 \pm 6.6$ \\
\hline Waist circumference $(\mathrm{cm})$ & $98.5 \pm 14.2$ & $99.1 \pm 16.1$ & $99.8 \pm 17.3$ & $<0.001$ & $99.1 \pm 15.9$ \\
\hline LBSIZ & $-0.01 \pm 1.01$ & $-0.01 \pm 1.00$ & $0.00 \pm 1.00$ & 0.736 & $-0.01 \pm 1.00$ \\
\hline Systolic BP (mmHg) & $122.6 \pm 19.7$ & $123.6 \pm 18.9$ & $127.2 \pm 20.8$ & $<0.001$ & $124.1 \pm 19.7$ \\
\hline Diastolic BP (mmHg) & $69.2 \pm 12.7$ & $69.7 \pm 12.8$ & $71.6 \pm 14.6$ & $<0.001$ & $70.0 \pm 13.2$ \\
\hline Hypertension & $4135(38.2 \%)$ & $8947(47.4 \%)$ & $4665(54.6 \%)$ & $<0.001$ & $17747(46.4 \%)$ \\
\hline FBG level (mg/dL) & $111.1 \pm 41.9$ & $105.0 \pm 29.7$ & $108.4 \pm 42.2$ & $<0.001$ & $107.5 \pm 36.5$ \\
\hline $\mathrm{HbA}_{1 \mathrm{c}}(\%)$ & $5.8 \pm 1.3$ & $5.5 \pm 0.8$ & $5.9 \pm 1.3$ & $<0.001$ & $5.7 \pm 1.1$ \\
\hline Diabetes Mellitus & $2175(19.3 \%)$ & $2636(13.6 \%)$ & $1795(21.0 \%)$ & $<0.001$ & $6606(16.9 \%)$ \\
\hline Total cholesterol (mg/dL) & $198.9 \pm 42.0$ & $198.8 \pm 43.3$ & $192.0 \pm 41.9$ & $<0.001$ & $197.4 \pm 42.7$ \\
\hline $\mathrm{TG}(\mathrm{mg} / \mathrm{dL})$ & $165.9 \pm 150.2$ & $150.5 \pm 130.0$ & $110.3 \pm 83.8$ & $<0.001$ & $146.4 \pm 129.8$ \\
\hline HDL-C (mg/dL) & $50.4 \pm 14.4$ & $53.3 \pm 16.6$ & $56.1 \pm 17.2$ & $<0.001$ & $53.0 \pm 16.3$ \\
\hline Dyslipidemia & $4458(52.9 \%)$ & $9070(54.6 \%)$ & $3373(48.3 \%)$ & $<0.001$ & $16901(52.8 \%)$ \\
\hline CVD & $837(7.2 \%)$ & $2548(12.9 \%)$ & $942(10.4 \%)$ & $<0.001$ & $4327(10.7 \%)$ \\
\hline
\end{tabular}

Table 1. Characteristics of the subjects according to ethnicity/race. Data are presented as the means $\pm S D$ or number (\%). Abbreviations: CVD, cardiovascular disease; BMI, body mass index; LBSIZ, z-score of the logtransformed A Body Shape Index; WC, waist circumference; BP, blood pressure; FBG, fasting blood glucose; HbA1c, haemoglobin A1c; HDL-C, high-density lipoprotein cholesterol; TG, triglyceride.

\begin{tabular}{|l|l|l|l|l|l|l|l|l|l|}
\hline \multirow{2}{*}{ Ethnicity/race } & \multicolumn{9}{|l|}{ Percentile } \\
\cline { 2 - 10 } & $\mathbf{1 0}^{\text {th }}$ & $\mathbf{2 0}^{\text {th }}$ & $\mathbf{3 0}^{\text {th }}$ & $\mathbf{4 0}^{\text {th }}$ & $\mathbf{5 0}^{\text {th }}$ & $\mathbf{6 0}^{\text {th }}$ & $\mathbf{7 0}^{\text {th }}$ & $\mathbf{8 0}^{\text {th }}$ & $\mathbf{9 0}^{\text {th }}$ \\
\hline Total & -1.30 & -0.84 & -0.52 & -0.24 & 0.02 & 0.26 & 0.53 & 0.84 & 1.26 \\
\hline Hispanic & -1.28 & -0.83 & -0.50 & -0.23 & 0.01 & 0.24 & 0.51 & 0.81 & 1.25 \\
\hline Non-Hispanic White & -1.31 & -0.84 & -0.53 & -0.25 & 0.01 & 0.27 & 0.54 & 0.85 & 1.27 \\
\hline Non-Hispanic Black & -1.30 & -0.85 & -0.52 & -0.24 & 0.04 & 0.29 & 0.55 & 0.85 & 1.26 \\
\hline
\end{tabular}

Table 2. Distribution of LBSIZ according to ethnicity/race. LBSIZ, z-score of the log-transformed A Body Shape Index.

\begin{tabular}{|l|c|l|}
\hline & FMI & ASMI \\
\cline { 2 - 3 } & $\boldsymbol{\beta}(\mathbf{9 5} \% \mathbf{C I})$ & $\boldsymbol{\beta}(\mathbf{9 5} \% \mathbf{C I})$ \\
\hline LBSIZ & $0.178(0.112,0.245)$ & $-0.221(-0.244,-0.198)$ \\
\hline WC & $0.223(0.221,0.225)$ & $0.060(0.059,0.061)$ \\
\hline BMI & $0.602(0.598,0.606)$ & $0.184(0.182,0.186)$ \\
\hline Conicity Index & $21.075(20.434,21.717)$ & $3.244(2.989,3.499)$ \\
\hline BRI & $1.720(1.703,1.736)$ & $0.454(0.445,0.463)$ \\
\hline CUN-BAE & $0.467(0.463,0.0 .470)$ & $0.146(0.144,0.148)$ \\
\hline New BMI & $0.586(0.583,0.590)$ & $0.178(0.176,0.180)$ \\
\hline WHtR & $37.794(37.440,38.148)$ & $10.036(9.836,10.236)$ \\
\hline
\end{tabular}

Table 3. Association of obesity parameters with FMI and ASMI. Linear regression analysis adjusted for age, sex and ethnicity/race. LBSIZ: z-score of the log-transformed A Body Shape Index; BMI: body mass index; WC: waist circumference; Body Roundness Index: BRI; Clinica Universidad de Navarra-Body Adiposity Estimator: CUN-BAE; waist to height ratio: WHtR.

according to ethnicity/race, the AUCs of LBSIZ were 0.680 (95\% CI: 0.661-0.699), 0.708 (95\% CI: 0.697-0.718), and 0.678 (95\% CI: 0.661-0.695) for the Hispanic, Non-Hispanic white, and Non-Hispanic black groups, respectively. The overall cut-off value of LBSIZ was $0.35\left(65^{\text {th }}\right.$ percentile, sensitivity, $63.3 \%$; specificity, $\left.66.6 \%\right)$. Its corresponding sensitivity and specificities by ethnicity/race were $62.4 \%$ and $66.2 \%, 64.6 \%$ and $67.4 \%$, and $60.7 \%$ and $65.3 \%$ for the Hispanic, Non-Hispanic white, and Non-Hispanic black groups, respectively. The OR for CVD, 
(a)

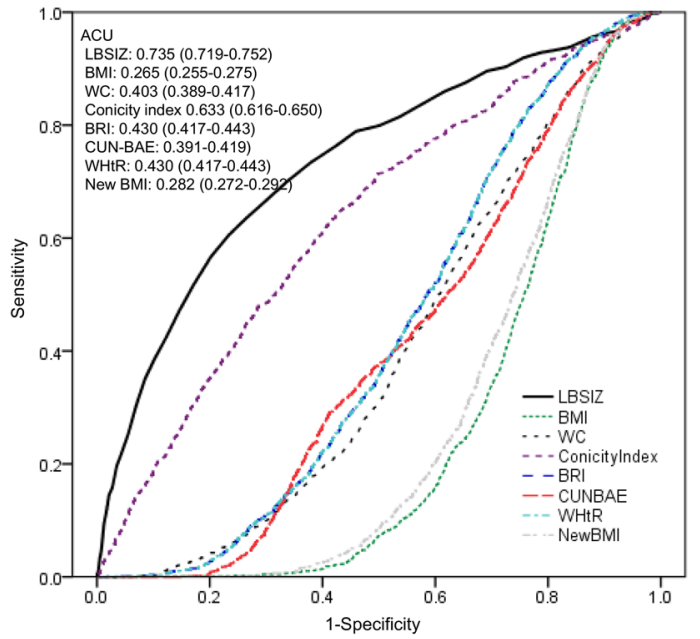

(b)

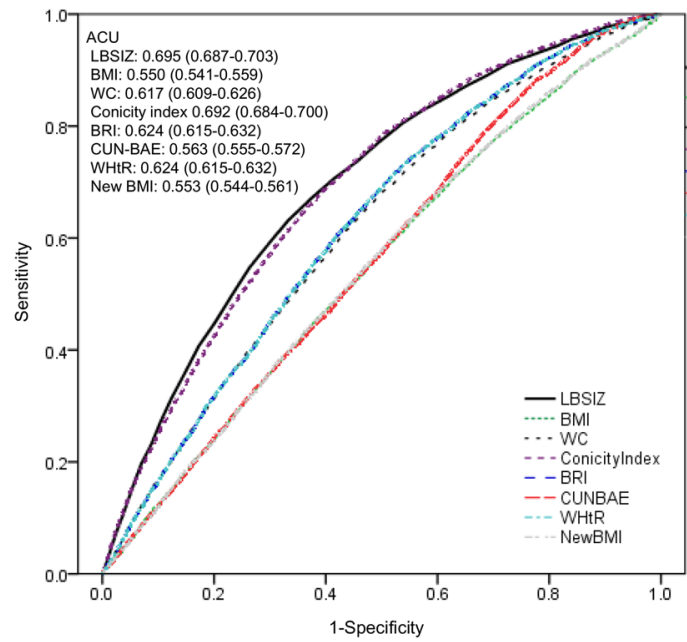

Figure 2. Receiver operating characteristic curves for CVD according to obesity parameters. (a) Sarcopenic obesity; (b) CVD. LBSIZ: z-score of the log-transformed A Body Shape Index; BMI: body mass index; WC: waist circumference; Body Roundness Index: BRI; Clinica Universidad de Navarra-Body Adiposity Estimator: CUN-BAE; waist to height ratio: WHtR; CVD: cardiovascular disease.

(a)

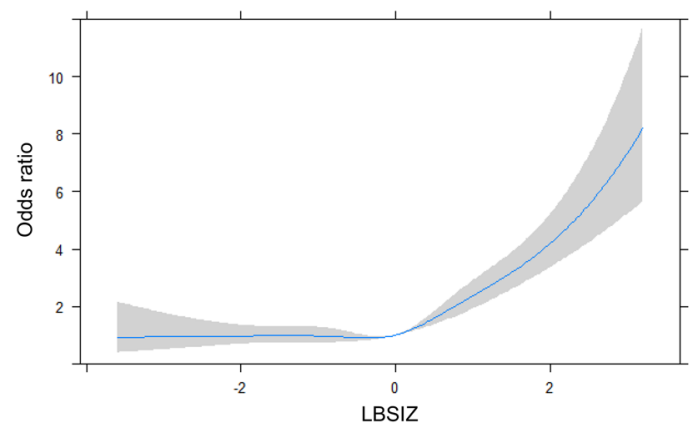

(b)

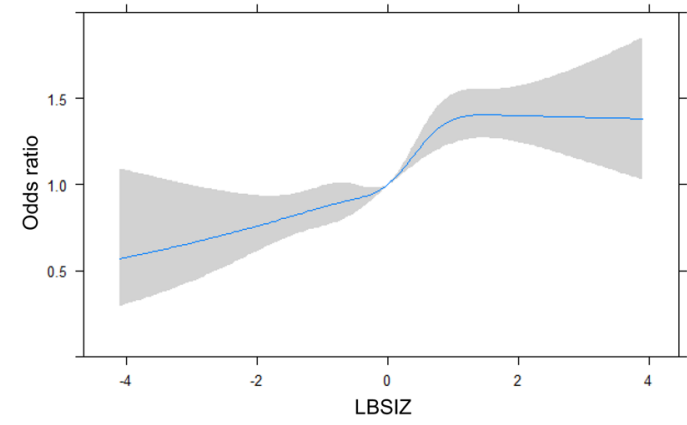

Figure 3. The odds ratio according to the continuous value of LBSIZ. (a) Sarcopenic obesity; (b) CVD. Adjusted for age, sex and ethnicity/race. LBSIZ: z-score of the log-transformed A Body Shape Index (LBSIZ).

after adjusting for age, sex, and ethnicity/race was 1.40 (95\% CI, 1.30-1.51) at the estimated cut-off value of LBSIZ. On the restricted cubic spline regression plot, ORs increased after the median (0) of LBSIZ (Fig. 3b).

\section{Discussion}

This study investigated the association of LBSIZ with both sarcopenic obesity and CVD, using a representative US sample, and found that LBISZ showed superior association thereto, compared with other weight-, and WC-related obesity measures. The study also provided appropriate cut-off values of LBSIZ, to be able to identify individuals at high risk for sarcopenic obesity or CVD, irrespective of sex and race, and to improve its clinical usefulness in practice.

A number of epidemiologic studies showed heterogeneous results regarding the association between traditional $\mathrm{BMI}$ and $\mathrm{CVD}^{10-12}$. This heterogeneity might be due to the limitations in defining obesity based on BMI, which does not differentiate fat from lean mass, nor does it consider the distribution of adipose tissue ${ }^{1,18-25}$. As central deposition of adipose tissues due to obesity became known to be a major cause of CVD-related mortality and morbidity, WC has emerged as an important complement to BMI, as an indicator of visceral adiposity, metabolic risk, and increased morbidity and mortality ${ }^{26-30}$. The National Cholesterol Education Program-Adult Treatment Panel III (NCEP-ATP III) criteria use WC instead of BMI to define metabolic syndrome ${ }^{31}$. However, there are insufficient data regarding the appropriate WC values to define obesity among the different age groups and sexes ${ }^{19,28,32}$. Due to these limitations of BMI and WC, many researchers have explored other obesity indices $^{33,34}$

In 2012, Krakauer et al. proposed a new obesity index, $\mathrm{ABSI}^{12}$, using the $\mathrm{WC}$, weight, and height data from the NHANES 1999-2004. Several studies reported that ABSI predicted premature mortality and CVD more 
effectively than did BMI or $\mathrm{WC}^{13,35,36}$. However, other studies reported that the role of ABSI has been challenged as a risk predictor of mortality, cardiovascular diseases, and metabolic syndrome ${ }^{32,37,38}$. A recent meta-analysis of 38 studies reported ambiguous results, where ABSI outperformed BMI and WC in predicting all-cause mortality, but underperformed in predicting hypertension, and diabetes mellitus ${ }^{39}$. In addition, several studies reported that ABSI had limitations in predicting fat mass ${ }^{40}$.

Although LBSIZ is a revised measure of abdominal obesity based on ABSI, it was better in predicting the development of CVD, than BMI or WC, and even improved the predictability of the Framingham risk score for CVD events in a prospective Korean cohor ${ }^{15}$. In another population-based study using the Korea NHANES data, LBSIZ showed a linear relationship with $\mathrm{CVD}^{17,41}$. Interestingly, this study showed that LBISZ has a superior association with CVD in a representative US sample, compared with other obesity parameters. Although the mechanism is unclear, we suspect that the association of obesity parameters with body composition might have played an important intermediary role thereto. In this regard, therefore, the result of LBSIZ having a positive association with FMI, and a negative association with ASMI, is consistent with the results of the previous Rotterdam study ${ }^{14}$. Conversely, other obesity parameters, associated positively with both fat mass and ASMI, could not identify sarcopenic obesity, or the presence of low muscle mass accompanied by a high fat mass ${ }^{42}$.

A complex (albeit not fully elucidated) interplay of several underlying mechanisms are responsible for the development of sarcopenic obesity; fat accumulation, which is related to the increase of proinflammatory cytokines, oxidative stress, and insulin resistance, might cause muscle fiber atrophy and mitochondrial dysfunction, leading to the development and progression of sarcopenia. Likewise, sarcopenia could worsen obesity through the decline of physical activity and energy expenditure, thereby resulting in further sarcopenia, leading to a vicious cycle of atrophy, aggravating their effects on metabolic, and functional abnormalities ${ }^{43,44}$. While a clear definition of sarcopenic obesity was not available, several studies showed that it was related to metabolic diseases and physical disability ${ }^{45-47}$; a few studies about the relationship between sarcopenic obesity and CVD, and mortality have been performed ${ }^{48-50}$. According to a recent meta-analysis, sarcopenic obesity was assessed to be associated with a $24 \%$ increased risk of all-cause mortality ${ }^{51}$. This study used Baumgartner's definition of sarcopenic obesity ${ }^{42}$, and showed that LBSIZ is the only measure of obesity related to sarcopenic obesity among all the obesity parameters that were considered. These results indicate the usefulness of LBSIZ in screening sarcopenic obesity, unlike other obesity measures.

LBSIZ has limitations in its use in clinical settings, or in epidemiological studies, considering the calculation of LBSIZ is highly complicated. Therefore, we present a simpler formula that can be used to estimate LBSIZ for each race, using a large-scale dataset from the NHANES 1999-2016. In addition, the estimated cut-off value can be used as a clinical standard, thereby facilitating easier clinical use, irrespective of sex or race. Considering the increase in ORs for sarcopenic obesity and CVD after the median LBSIZ in the restricted cubic spline regression plots, the cut-off value corresponding to the $65^{\text {th }}$ percentile of LBSIZ can effectively help to identify individuals at a high risk of sarcopenic obesity and CVD.

Despite our interesting findings, there are a few limitations in this study. First, this was a cross-sectional study, and therefore, the causal relationship between obesity and CVD remains to be examined further, using cohort data. Secondly, this study did not analyze mortality, and may have missed fatal CVD events due to a lack of data. Third, we only examined weight-, and WC-related obesity measures, omitting hip circumference-related measures due to data availability. Finally, potential confounders are yet to be further examined to elucidate the LBSIZ-related pathophysiological mechanism.

In conclusion, LBSIZ showed a stronger association with both sarcopenic obesity and CVD, compared with other obesity parameters. These results may be useful when conducting a CVD risk assessment in clinical settings; the proposed LBSIZ cut-off values have the potential to be a useful clinical standard.

\section{Materials and Methods}

Study population. Data were collected from the NHANES dataset between 1999 and 2016. Exclusion criteria were as follows: those aged $\leq 20$ years, those with missing data (CVD questionnaire, anthropometric, or laboratory data), or those who were not Hispanic, non-Hispanic whites, or non-Hispanic blacks. Finally, 40,468 of 92,062 participants were included in this study (Fig. 1). Because the DEXA data were available between 1999 and $2005,11,780$ participants were included in the subgroup analysis for body composition.

Measurements of obesity parameters and body composition. WC was measured using a measuring tape at the upper-lateral border of the iliac $\mathrm{crest}^{52}$. BMI was defined by the weight in kilograms, divided by the height in meters squared $\left(\mathrm{kg} / \mathrm{m}^{2}\right)$. Conicity Index, BRI, CUN-BAE, new BMI, and WHtR were calculated based on the earlier-suggested formula ${ }^{13,32,53,54}$

Conicity Index: WC(m)/[0.1093 sqrt(Weight(kg)/Height(m))]

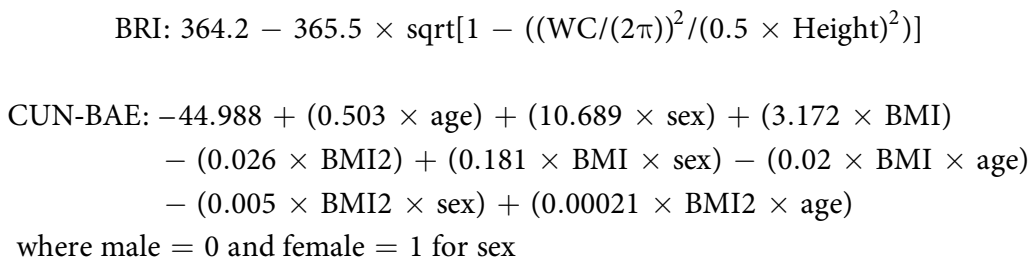




\author{
New BMI: $1.3 \times\left(\right.$ Weight $(\mathrm{kg}) /$ Height $\left.(\mathrm{m})^{2}\right)$
}

\title{
WHtR: WC(cm)/Height $(\mathrm{cm})$
}

We calculated the LBSIZ based on the regression $\left[\ln (\right.$ waist $)=a_{0}+a_{1} \ln ($ weight $)+a_{2} \ln ($ height $\left.)+\delta\right]$, standardizing the waist values according to weight and height. After respective estimation for each race, the log-transformed ABSI (LBSI) was calculated using the following equation: $\log \left[\right.$ waist $/\left(\exp \left(\mathrm{a}_{0}\right) \times\right.$ weight $^{\mathrm{al}} \times$ height $^{\mathrm{a}}{ }^{2}$. In the next step, the z-score of the log-transformed ABSI (LBSIZ) was calculated using the mean of the LBSI and standard deviation (SD) of LBSI: LBSIZ = (LBSI-mean(LBSI))/SD(LBSI). More details are provided in the supplementary Excel file, and are referenced in a previous study ${ }^{16}$.

Assessment of body composition was performed by whole body DEXA using a Hologic QDR $4500 \mathrm{~A}$ fan beam X-ray bone densitometer (Hologic Inc., Marlborough, MA, USA). All DEXA scans were analyzed using Hologic Discovery software (version 12.1, Hologic Inc.) to measure total and regional body composition, including bone mineral content lean body mass, fat mass, and \% body fat. ASM was defined as the sum of the total lean mass of both the arms and legs. ASM index was defined as ASM divided by the square of the height. Fat mass index was defined as the total fat mass divided by the square of the height.

Definition of sarcopenic obesity and CVD. Sarcopenic obesity was defined as ASMI $<7.26 \mathrm{~kg} / \mathrm{m}^{2}$, and $\%$ body fat $>27 \%$ in men, or ASMI $<5.45 \mathrm{~kg} / \mathrm{m}^{2}$, and $\%$ body fat $>38 \%$ in women, based on the definitions by Baumgartner ${ }^{42}$

A structured questionnaire was used to investigate CVD. A patient was deemed to have CVD if they had at least one of the following conditions: angina pectoris, coronary heart disease, myocardial infarction, congestive heart failure, or cerebrovascular disease $\mathrm{e}^{55}$.

Statistical analysis. For summary statistics, we presented the mean with a $95 \%$ CI, or prevalence (\%) according to ethnicity/race. Continuous variables were assessed using one-way analysis of variance, and categorical variables were assessed using the Pearson's chi-square test. We estimated the distribution of LBSIZ, and verified the correlations between the obesity indices. A receiver operating characteristic (ROC) curve was used to analyze the correlation between sarcopenic obesity or CVD, and each of the obesity parameters, and the de Long's test was used to identify obesity indices that were significantly superior ${ }^{56}$. We validated the model using 1,000 samples generated through the bootstrap method. The cut-off value of LBSIZ was determined as the value with the highest Youden's index, conditional on having a sensitivity and specificity greater than $60 \%{ }^{57}$. A multivariate logistic regression analysis was performed to determine the OR for sarcopenic obesity and CVD. Furthermore, the OR of LBSIZ for sarcopenic obesity and CVD was analyzed using restricted cubic spline splits with five knots. The analyses were performed using SPSS software version 24.0 (IBM Inc., Armonk, NY, USA), R (version 3.1.0, R Foundation for Statistical Computing, Vienna, Austria) and Stata version 15 (Stata Corporation, College Station, Texas, USA). A P-value of 0.05 was considered statistically significant.

Ethics statement. The study protocol was approved by the institutional review board of Kangnam Sacred Heart Hospital (IRB No. HKS 2017-07-007). All participants volunteered, and provided written informed consent prior to their enrolment. All participants' records were anonymized before being accessed by the authors. All methods were carried out in accordance with the approved guidelines and regulations.

\section{References}

1. World Health Organization. Fact sheet on obesity and overweight. [accessed 2018 May 13], Available from, http://www.who.int/en/ news-room/fact-sheets/detail/obesity-and-overweight (2017).

2. Solomon, C. G. \& Manson, J. E. Obesity and mortality: a review of the epidemiologic data. Am J Clin Nutr. 66, 1044S-1050S (1997).

3. Krauss, R. M., Winston, M., Fletcher, B. J. \& Grundy, S. M. Obesity: impact on cardiovascular disease. Circulation. 98, 1472-1476 (1998).

4. Moon, S. et al. The influence of physical activity on risk of cardiovascular disease in people who are obese but metabolically healthy. PLoS One. 12, e0185127 (2017).

5. Wallstrom, P., Bjartell, A., Gullberg, B., Olsson, H. \& Wirfalt, E. A prospective Swedish study on body size, body composition, diabetes, and prostate cancer risk. Brit J Cancer. 100, 1799-1805 (2009).

6. World Health Organization. Global Health Risks: Mortality and Burden of Disease Attributable to Selected Major Risks (WHO Press, 2009).

7. Batsis, J. A. \& Villareal, D. T. Sarcopenic obesity in older adults: aetiology, epidemiology and treatment strategies. Nat Rev Endocrinol. 14, 513-537 (2018).

8. Kushner, R. F. Clinical assessment and management of adult obesity. Circulation. 126, 2870-2877 (2012).

9. Heymsfield, S. B., Scherzer, R., Pietrobelli, A., Lewis, C. E. \& Grunfeld, C. Body Mass Index as a phenotypic expression of adiposity: quantitative contribution of muscularity in a population-based sample. Int J Obes (Lond). 33, 1363-1373 (2009).

10. Romero-Corral, A. et al. Accuracy of body mass index in diagnosing obesity in the adult general population. Int J Obes (Lond). 32, 959-966 (2008)

11. Schneider, H. J. et al. The predictive value of different measures of obesity for incident cardiovascular events and mortality. J Clin Endocrinol Metab. 95, 1777-1785 (2010).

12. Flegal, K. M., Graubard, B. I., Williamson, D. F. \& Gail, M. H. Excess deaths associated with underweight, overweight, and obesity. JAMA. 293, 1861-1867 (2005).

13. Krakauer, N. Y. \& Krakauer, J. C. A New body shape index predicts mortality hazard independently of body mass index. PLoS ONE. 7, e39504 (2012).

14. Dhana, K. C. et al. Association of anthropometric measures with fat and fat-free mass in the elderly: the Rotterdam study. Maturitas. 88, 96-100 (2016). 
15. Chung, W., Park, C. G. \& Ryu, O. H. Association of a New Measure of Obesity with Hypertension and Health-Related Quality of Life. PLoS One. 11, e0155399 (2016).

16. Moon, S. et al. Effectiveness of Z-score of log-transformed A Body Shape Index (LBSIZ) in predicting cardiovascular disease in Korea: the Korean Genome and Epidemiology Study. Sci Rep. 8, 12094 (2018).

17. Chung, W. et al. The Association between Z-Score of Log-Transformed A Body Shape Index and Cardiovascular Disease in Korea. Diabetes Metab J. 43, e34 (2019).

18. Rahman, M. \& Berenson, A. B. Accuracy of current body mass index obesity classification for white, black and Hispanic reproductive-age women. Obstet Gynecol. 115, 982 (2010).

19. Gurunathan, U. \& Myles, P. S. Limitations of body mass index as an obesity measure of perioperative risk. Br J Anaesth. 116, 319-321 (2016).

20. Rothman, K. J. BMI-related errors in the measurement of obesity. Int J Obes 32, 56-59 (2008).

21. Coutinho, T. et al. Combining body mass index with measures of central obesity in the assessment of mortality in subjects with coronary disease: role of "normal weight central obesity". J Am Coll Cardiol. 61, 553-560 (2013).

22. Gastaldelli, A. et al. Metabolic effects of visceral fat accumulation in type 2 diabetes. J Clin Endocrinol Metab. 87, 5098-5103 (2002).

23. Donohoe, C. L., Doyle, S. L. \& Reynolds, J. V. Visceral adiposity, insulin resistance and cancer risk. Diabetol Metab Syndr. 3, 12 (2011).

24. Shimizu, I., Yoshida, Y. \& Minamino, T. Maintenance of Subcutaneous Fat Homeostasis Improves Systemic Metabolic Dysfunction in Obesity. Diabetes. 64, 3984-3986 (2015).

25. Tran, T. T., Yamamoto, Y., Gesta, S. \& Kahn, C. R. Beneficial effects of subcutaneous fat transplantation on metabolism. Cell Metab. 7, 410-420 (2008)

26. Van Gaal, L. F., Mertens, I. L. \& De Block, C. E. Mechanisms linking obesity with cardiovascular disease. Nature. 444, 875-880 (2006).

27. Din-Dzietham, R., Liu, Y., Bielo, M. V. \& Shamsa, F. High blood pressure trends in children and adolescents in national Surveys, 1963 to 2002. Circulation. 116, 1488-1496 (2007).

28. Onat, A. et al. Measures of abdominal obesity assessed for visceral adiposity and relation to coronary risk. Int J Obes Relat Metab Disord. 28, 1018-1025 (2004).

29. Bays, H. Central obesity as a clinical marker of adiposopathy; increased viscera; adiposity as a surrogate marker for global fat dysfunction. Curr Opin Endocrinol Diabetes Obes. 21, 345-351 (2014).

30. Chan, J. M. et al. Obesity, fat distribution, and weight gain as risk factors for clinical diabetes in men. Diabetes Care 17, 961-969 (1994).

31. Grundy, S. M. et al. Diagnosis and management of the metabolic syndrome: an American Heart Association/National Heart, Lung and Blood Institute scientific statement. Circulation 112, 2735-2752 (2005).

32. Amirabdollahian, F. \& Haghighatdoost, F. Anthropometric Indicators of Adiposity Related to Body Weight and Body Shape as Cardiometabolic Risk Predictors in British Young Adults: Superiority of Waist-to-Height Ratio. J Obes 2018, 8370304 (2018).

33. Bener, A. et al. Obesity index that better predict metabolic syndrome: body mass index, waist circumference, waist hip ratio, or waist height ratio. J Obes 2013, 269038 (2013).

34. Janssen, I., Katzmarzyk, P. T. \& Ross, R. Waist circumference and not body mass index explains obesityrelated health risk. Am J Clin Nutr 79, 379-384 (2004).

35. Lee, D. Y., Lee, M. Y. \& Sung, K. C. Prediction of Mortality with A Body Shape Index in Young Asians: Comparison with Body Mass Index and Waist Circumference. Obesity (Silver Spring). 26, 1096-1103 (2018).

36. Bozorgmanesh, M. et al. CVD-predictive performances of "a body shape index" versus simple anthropometric measures: Tehran lipid and glucose study. Eur J Nutr. 55, 147-157 (2016).

37. Haghighatdoost, F. et al. Assessing body shape index as a risk predictor for cardiovascular diseases and metabolic syndrome among Iranian adults. Nutrition 30, 636-644 (2014).

38. He, S., Zheng, Y., Wang, H. \& Chen, X. Assessing the relationship between a body shape index and mortality in a group of middleaged men. Clin Nutr 36, 1355-1359 (2017).

39. Ji, M., Zhang, S. \& An, R. Effectiveness of A Body Shape Index (ABSI) in predicting chronic diseases and mortality: a systematic review and meta-analysis. Obes Rev. 19, 737-759 (2018).

40. Santos, D. A. et al. Utility of novel body indices in predicting fat mass in elite athletes. Nutrition 31, 948-54 (2015).

41. Moon, S. et al. Association of Z-Score of the Log-Transformed A Body Shape Index with Cardiovascular Disease in People Who Are Obese but Metabolically Healthy: The Korea National Health and Nutrition Examination Survey 2007-2010. Journal of Obesity \& Metabolic Syndrome 27, 158-165 (2018).

42. Baumgartner, R. N. et al. Sarcopenic obesity predicts instrumental activities of daily living disability in the elderly. Obes Res 12, 1995-2004 (2004).

43. Kalinkovich, A. \& Livshits, G. Sarcopenic obesity or obese sarcopenia: A cross talk between age-associated adipose tissue and skeletal muscle inflammation as a main mechanism of the pathogenesis. Ageing Res Rev 35, 200-221 (2017).

44. Choi, K. M. Sarcopenia and sarcopenic obesity. Korean J Intern Med. 31, 1054-1060 (2016).

45. Kohara, K. Sarcopenic obesity in aging population: current status and future directions for research. Endocrine 45, 15-25 (2014).

46. Stenholm, S. et al. Sarcopenic obesity: definition, cause and consequences. Curr Opin Clin Nutr Metab Care 11, 693-700 (2008).

47. Rolland, Y. et al. Difficulties with physical function associated with obesity, sarcopenia, and sarcopenic-obesity in communitydwelling elderly women: the EPIDOS (EPIDemiologie de l'OSteoporose) Study. Am. J. Clin. Nutr 89, 1895-1900 (2009).

48. Wannamethee, S. G., Shaper, A. G., Lennon, L. \& Whincup, P. H. Decreased muscle mass and increased central adiposity are independently related to mortality in older men. Am J Clin Nutr 86, 1339-1346 (2007).

49. Stephen, W. C. \& Janssen, I. Sarcopenic-obesity and cardiovascular disease risk in the elderly. J Nutr Health Aging 13, 460-466 (2009).

50. Batsis, J. A., Mackenzie, T. A., Barre, L. K., Lopez-Jimenez, F. \& Bartels, S. J. Sarcopenia, sarcopenic obesity and mortality in older adults: results from the National Health and Nutrition Examination Survey III. Eur J Clin Nutr 68, 1001-1007 (2014),

51. Tian, S. \& Xu, Y. Association of sarcopenic obesity with the risk of all-cause mortality: a meta-analysis of prospective cohort studies. Geriatr Gerontol Int 16, 155-166 (2016).

52. Centers for Disease Control and Prevention Anthropometric Procedures manual. Available from: URL: https://wwwn.cdc.gov/nchs/ data/nhanes/2011-2012/manuals/anthropometry_procedures_manual.pdf [accessed $2018 \mathrm{Feb} 20$ ]

53. Motamed, N. et al. Conicity Index and Waist-to-Hip Ratio Are Superior Obesity Indices in Predicting 10-Year Cardiovascular Risk Among Men and Women. Clin Cardiol 38, 527-534 (2015).

54. Thomas, D. M. et al. Relationships between body roundness with body fat and visceral adipose tissue emerging from a new geometrical model. Obesity 21, 2264-2271 (2013).

55. Arnlöv, J., Ingelsson, E., Sundström, J. \& Lind, L. Impact of body mass index and the metabolic syndrome on the risk of cardiovascular disease and death in middle-aged men. Circulation. 19(121), 230-6 (2010).

56. DeLong, E. R., DeLong, D. M. \& Clarke-Pearson, D. L. Comparing the Areas Under Two or More Correlated Receiver Operating Characteristics Curves: A Nonparametric Approach. Biometrics 44, 837-845 (1988)

57. Youden, W. J. Index for rating diagnostic tests. Cancer. 3, 32-35 (1950). 


\section{Author Contributions}

Moon, S., Kim, D.S. and Chung, W. contributed to the research design; Moon, S., Chung, W., Yu J.M. Chung, H.S. \& Park, J.H. participated in the design and performance of the research and data analysis; Moon, S., Kim, D.S., and Chung, W. wrote the main manuscript text and Moon, S. prepared Figures 1-3. All authors reviewed the manuscript.

\section{Additional Information}

Supplementary information accompanies this paper at https://doi.org/10.1038/s41598-019-45717-8.

Competing Interests: The authors declare no competing interests.

Publisher's note: Springer Nature remains neutral with regard to jurisdictional claims in published maps and institutional affiliations.

(c) (i) Open Access This article is licensed under a Creative Commons Attribution 4.0 International License, which permits use, sharing, adaptation, distribution and reproduction in any medium or format, as long as you give appropriate credit to the original author(s) and the source, provide a link to the Creative Commons license, and indicate if changes were made. The images or other third party material in this article are included in the article's Creative Commons license, unless indicated otherwise in a credit line to the material. If material is not included in the article's Creative Commons license and your intended use is not permitted by statutory regulation or exceeds the permitted use, you will need to obtain permission directly from the copyright holder. To view a copy of this license, visit http://creativecommons.org/licenses/by/4.0/.

(C) The Author(s) 2019 